NASA Technical Memorandum 101085

\title{
Piloted Simulation of a Ground-Based Time-Control Concept for Air Traffic Control
}

Thomas J. Davis and Steven M. Green

June 1989

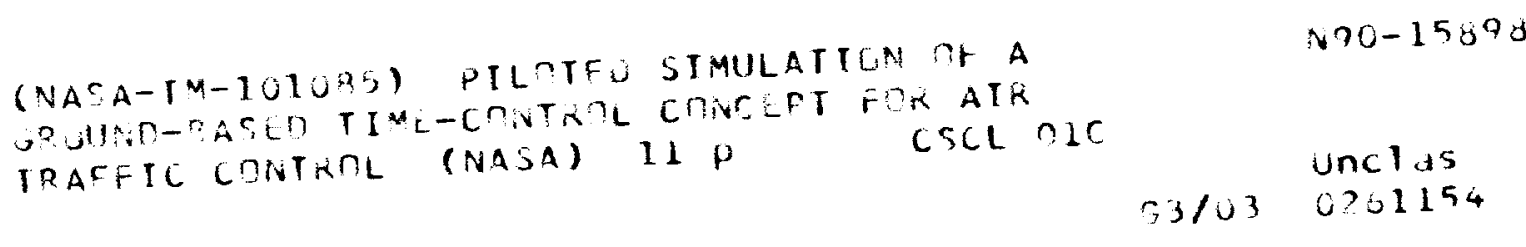




\author{
ERRATA \\ Piloted Simulation of a \\ Ground-Based Time-Control \\ Concept for Air Traffic Control \\ Thomas J. Davis and Steven M. Green \\ June 1989
}

This NASA Technical Memorandum was erroneously issued as TM 101086. The correct number is NASA Technical Memorandum 101085.

Please change the number as it appears at the top of the cover, the top of the title page, and in block 1 of the Report Documentation Page (the last page of the report) to read 101085 .

Issued $1 / 19 / 90$ 

NASA Technical Memorandum 101085

\section{Piloted Simulation of a Ground-Based Time-Control Concept for Air Traffic Control}

Thomas J. Davis and Steven M. Green, Ames Research Center, Moffett Field, California

June 1989

\section{N/SN}

National Aeronautics and

Space Administration 


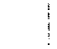




\title{
PILOTED SIMULATION OF A GROUND-BASED TIME-CONTROL CONCEPT FOR AIR TRAFFIC CONTROL
}

\author{
Thomas J. Davis and Steven M. Green \\ NASA Ames Research Center \\ Moffett Field, CA
}

\begin{abstract}
A concept for aiding air traffic controllers in efficiently spacing traffic and meeting scheduled arrival times at a metering fix has been developed and tested in a real-time simulation. The automation aid, referred to as the ground-based fourdimensional descent advisor (DA), is based on accurate models of aircraft performance and weather conditions. The DA generates suggested clearances, including both top-of-descent-point and speed-profile data, for one or more aircraft in order to achieve specific time or distance separation objectives. The DA algorithm is used by the air traffic controller to resolve conflicts and issue advisories to arrival aircraft. A joint simulation was conducted using a piloted simulator and an advanced-concept air traffic control simulation to study the acceptability and accuracy of the DA automation aid from both the pilot's and the air traffic controller's perspectives. This paper focuses on the results of the piloted simulation. In the piloted simulation, airline crews executed controller-issued descent advisories along standard curved-path arrival routes, and were able to achieve an arrival-time precision of $\pm 20 \mathrm{sec}$ at the metering fix. An analysis of errors generated in turns resulted in further enhancements of the algorithm to improve the predictive accuracy. Evaluations by pilots indicate general support for the concept and provide specific recommendations for improvement. Operational issues concerning how the DA was used for prediction, intrail spacing, and metering in a multiaircraft environment are described in a companion paper. (See Tobias, this conf.)
\end{abstract}

\section{INTRODUCTION}

In the past several years, the nation's air traffic control (ATC) system has become increasingly congested, delays have become common, and controller workload has increased. NASA Ames and other research laboratories are investigating methods for increasing the efficiency of terminal-area traffic management and decreasing controller workload. The current work focuses on the potential for using time-based trafficmanagement techniques. The success of this approach is dependent on its ability to handle aircraft regardless of the level of sophistication of the on-board equipment. Some new commercial aircraft are equipped with flightpath-management systems that are capable of generating and flying fourdimensional (4D) trajectories. Although these on-board systems will be an essential component of a time-based traffic management system, there will be a long transition period in which both equipped and unequipped aircraft will be flying. During this transition period, the success of time-based traffic management will be determined by how well controllers can control the arrival times of unequipped aircraft.

Copyright 1989 by the American Institute of Aeronautics and Astronautics, Inc. No copyright is asserted in the United States under Title 17, U.S. Code. The U.S. Government has a royalty-free license to exercise all rights under the copyright claimed herein for Governmental purposes. All other rights are reserved by the copyright owner.
The descent advisor (DA) is an automation tool that assists air traffic controllers in meeting arrival-time and spacing requirements for inbound traffic. The DA algorithm resides in a microprocessor-based workstation that interfaces with and receives aircraft surveillance data from the National Airspace Host Computer. As an unequipped aircraft approaches the descent area, the algorithm predicts the arrival time, taking into account the aircraft's performance capability, current wind and weather conditions, and the airline's standard operating procedure. The predicted arrival time is presented to the controllers, along with predictions for all other aircraft in their sector, by several graphical techniques. The controller chooses an optimum arrival time for an aircraft by adjusting the aircraft's descent-speed profile, using a mouse-based, menu-driven interface with the DA algorithm. When the controller accepts a descent-speed profile that will allow the aircraft to arrive at the desired time, the controller issues the speed profile advisory to the aircraft in the form of a clearance. A detailed description of the graphical controller interface for the DA algorithm is given in reference (1).

For the work described in this paper, a piloted simulator was used in conjunction with an ATC simulation to evaluate the performance of the ground-based, 4D, DA algorithm for controlling the arrival time of conventional (unequipped) aircraft. Arrival time is controlled to a position about 30 n.mi. from the airport, referred to as the metering fix or feeder fix. This is an intermediate point between cruise and touchdown where commercial jet traffic undergoes a transition from enroute descent to terminal area operation. The desired arrivaltime accuracy for unequipped aircraft at the feeder fix is \pm 20 sec.

An earlier study evaluated the DA performance for a single aircraft executing straight-in descents (2). Although the DA was shown to have considerable promise, pilots felt it was necessary to conduct an evaluation in an operational ATC environment with other traffic. In the current study, the piloted simulation was conducted in conjunction with a controller evaluation of the DA tool (3), to determine the precision with which airline pilots could fly curved-path, advisor-assisted descents in a realistic ATC environment. Additional evaluations included the effects of different wind conditions on pilot performance, and procedures for advisor-assisted route intercepts during descents. This paper first briefly reviews the 4D, DA algorithm and then describes the results of the piloted simulation studies.

\section{ALGORITHM DESCRIPTION}

In broad outline, the DA algorithm synthesizes a $4 D$ trajectory in the following way. First, the DA predicts the arrival time of an aircraft following a defined arrival route, based on the aircraft's standard operating procedures. Next, the DA computes a range of arrival times based on the aircraft's speed envelope. If the desired time, i.e., the time designated by ATC, is out of this range, a combination of speed change and path 
stretching is used to synthesize a $4 D$ trajectory. If the desired arrival time is within range, a 4D trajectory is determined by varying speed alone. Once the arrival path is defined, the algorithm iterates on descent speed until it computes a trajectory which meets the desired arrival time. For each iteration, the corresponding trajectory is calculated by integrating the aircraft's equations of motion backward from the conditions at the feeder fix to the conditions at the aircraft's current position. Finally, the DA translates the desired trajectory into an advisory consisting of a position to begin the descent procedure and the descent speed.

\section{Descent Procedure}

The trajectories generated by the DA are based upon a model of fuel-conservative descent procedures currently used in airline operations. This model uses a near-idle thrust descent with a constant Mach/calibrated airspeed (CAS) profile. Typical enroute descents are characterized by three distinct segments: the beginning of the descent procedure, a descent at a constant speed, and a level-off. At the beginning of the descent, the pilot normally throttles back to near-idle and then commands a pitch-down attitude to maintain the cruise Mach number. Under time control, however, the pilot may have to accelerate or decelerate to capture the specified descent speed. In the case of an acceleration, the pilot maintains cruise thrust during the descent until the descent speed is achieved. In the case of a deceleration, the pilot maintains cruise altitude, at idle thrust, until the descent speed is achieved, and then pitches over. During the next segment of the descent, the pilot flies a constant-speed profile (constant CAS with a constant Mach at the higher altitudes, if appropriate). Finally, as the aircraft approaches the desired altitude, the pilot reduces the descent rate to attain that altitude and then increases throttle to maintain the desired speed. For the case of a speed restriction at the level-off altitude, the pilot uses the level-off flight segment to decelerate before adding power.

To reduce fuel consumption, airlines prefer to descend at idle thrust and to avoid extended level flight at low altitude. Some situations (such as inclement weather requiring de-icing or turbulence penetration) require a minimum level of thrust; also, some of the older pressurization systems require a minimum level of thrust for smooth operation and passenger comfort. But in general, pilots attempt to descend at idle. For the purposes of this paper, the descent is modeled as being flown with idle thrust.

\section{DA Trajectory Model}

For each descent speed profile selected in the iteration process, the corresponding descent trajectory is computed by integrating a set of point mass equations of motion along the route of arrival. The equations are derived with respect to an Earthfixed reference frame using a rectangular coordinate system based upon a flat-Earth approximation. The trajectory is projected onto two planes - the horizontal, which defines the horizontal path, and the vertical, which defines the altitude profile along the path. The altitude profile and horizontal path are illustrated in Figures 1 and 2. This paper will focus on the horizontal plane, whereas previous papers on the DA have concentrated on the vertical plane profile only. A comprehensive description of the vertical trajectory synthesis may be found in references (2) and (4); however, since the variables

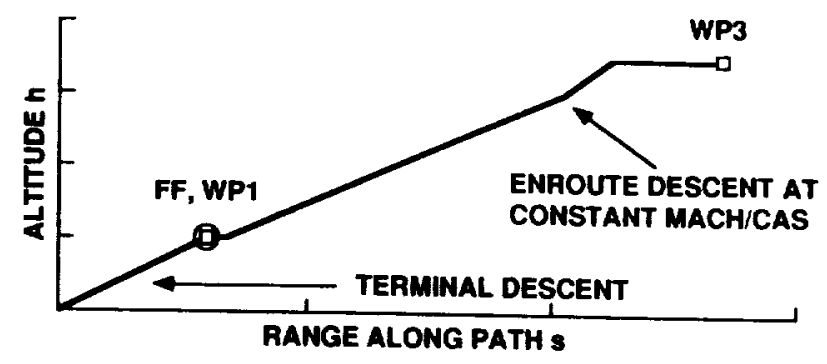

Fig. 1 Altitude profile of DA trajectory model.

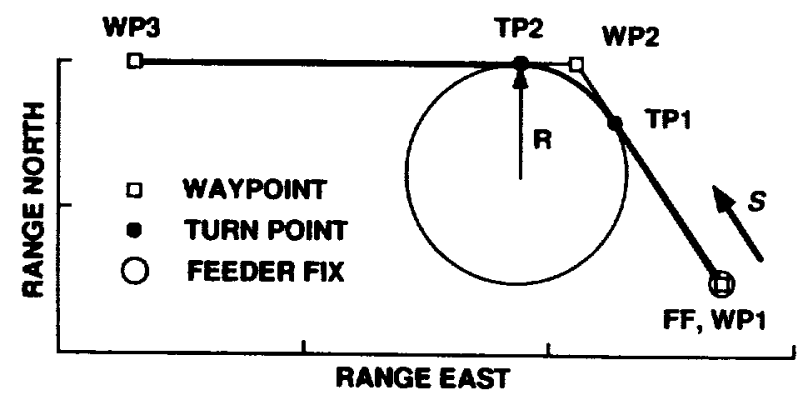

Fig. 2 Horizontal path of DA trajectory model.

describing motion in both planes are coupled, some discussion of the vertical profile is necessary here.

The coordinates $s$ and $h$ are defined as distance along the path and altitude, respectively. The kinematic equations to be integrated are

$$
\begin{gathered}
u=\frac{d s}{d t}=V_{t} \cos \left(\gamma_{a}\right)+U_{w s} \\
w \equiv \frac{d h}{d t}=V_{t} \sin \left(\gamma_{a}\right)
\end{gathered}
$$

where $u$ and $w$ are defined as the components of inertial velocity in the direction of $s$ and $h$, respectively, $V_{t}$ is the true airspeed, $\gamma_{a}$ is the aerodynamic flightpath angle, and $U_{w s}$ is the effective windspeed in the flightpath direction. The effective windspeed is defined as the difference between the groundspeed and the true airspeed. Assuming that the wind is known as a function of $s$ and $h$, equations (1) and (2) may be integrated once expressions for $V_{t}$ and $\gamma_{a}$ are known. The relationships for $V_{t}$ and $\gamma_{a}$ in terms of thrust, drag, weight, and Mach number or CAS are described in reference (2). The determination of thrust, drag, weight, and winds aloft will be discussed in a later section on algorithm implementation.

In its current implementation on a high-performance workstation, the DA algorithm contains models of two aircraft types, a Boeing 727-200 and a Boeing 737-100. However, the software is structured to accommodate any number of different aircraft types. The performance model, which includes detailed propulsive and aerodynamic information, is used to evaluate the thrust and drag terms just discussed. The propulsive model represents thrust as a function of engine pressure ratio (EPR), Mach number, temperature, and pressure. The thrustmanagement model defines either the EPR or the thrust value 
required during the descent for a particular thrust-management procedure. The Mach number is determined by the speed profile along with temperature, and the temperature and pressure are determined from atmospheric data. The aerodynamic model represents the drag coefficient as a function of lift coefficient. Mach number, and control-surface deflection (speed brake, flaps, and gear). Here, the lift coefficient is determined by using the approximation that lift is equal to weight. The control-surface deflection schedule is based upon speed and position. For the purposes of this paper, the aircraft is assumed to be in a clean configuration.

With regard to the modeling of thrust management during a descent, three cases have been identified. The first case is that of a constant-thrust setting. The second and third cases involve the variation of thrust to maintain a constant rate of descent and a constant inertial flightpath angle, respectively. The actual implementation of the algorithm allows for the assignment of any one of these cases to each distinct segment in a descent (e.g., constant Mach number, constant CAS, and so on) along with the corresponding descent rate or flightpath angle for the latter cases. Although a pilot would not actually fly a constant inertial flightpath angle, the combination of the three cases allows for the greatest flexibility in modeling automatic and manual descent procedures.

For each aircraft handled, the DA stores data detailing the synthesized 4D trajectory for later comparison with the aircraft's actual trajectory. This information is used to track time error (defined as the difference between the actual and desired schedules) which may grow during the descent. An example of a synthesized trajectory is given in reference (4).

\section{Horizontal Path}

The horizontal path is modeled as a series of straight-line segments connected by circular arcs, as illustrated in Figure 2. The line segments are defined by waypoints (e.g., WP1 and WP2), and the turns are defined by turn points (e.g., TP1 and TP2). The final waypoint used in generating a AD trajectory is the feeder fix. The radius of curvature, $R$, for each arc is determined as a function of the predicted average groundspeed throughout the turn. It is assumed that the turn is performed at an average altitude and true airspeed, which are approximated by the altitude and true airspeed of the aircraft when it is abeam of the turn intersection (e.g., WP2). The average true airspeed and effective windspeed are combined to predict the groundspeed throughout the turn.

Once the turn radius fixes the horizontal path for a particular set of conditions (e.g., speed profile and winds), the altitude profile is found by integrating equations (1) and (2) along the path. The approximations used above greatly simplify the coupled relationship between the horizontal path and the altitude profile, while still allowing the size of the turn to be determined as a function of airspeed, altitude, winds aloft, and course.

\section{SIMULATION DESCRIPTION}

A piloted simulation was conducted at the NASA Ames Research Center's Man-Vehicle Systems Research Facility
(MVSRF) 727-200 full-mission simulator to evaluate both pilot performance and acceptance of the DA clearances. The simulator, which is FAA-certified phase II, has a six-degree-offreedom motion system and a night/dusk computer-generatedimagery visual system. To generate a substantial and representative statistical data base, 42 airline pilots, from four major U.S. carriers, and 9 air traffic controllers participated in the evaluation.

The piloted simulation was conducted jointly with an ATC simulation of the DA/controller interface system. The simulation scenario was based on the Denver Air Route Traffic Control Center's (ATRCC) northwest arrival sectors. The piloted 727 simulator was one of several simulated aircraft participating in the study. The other aircraft were computer-generated pseudo-aircraft. The pseudo-aircraft are "flown" by pseudopilots who use a keyboard to initiate changes in aircraft altitude, speed, and heading. The commands are sent through a communications link to the pseudo-aircraft dynamics model which provides data for all pseudo-aircraft to the communications manager. The communications manager gathers data from all sources (pseudo-aircraft and simulated aircraft) and merges the data into a common format for use by the air traffic controller's display software. The air traffic controllers' objective in this evaluation was to meet a precise spacing requirement of 10 n.mi. intrail at a feeder fix. The DA was used to assist the controllers in formulating a strategy to achieve the spacing goal (see reference (3) for a more complete description).

In each 4-hr session, which typically consisted of five or six descents of 30 - to 40 -min duration each, two pilots (a captain and a first officer) alternated flying the descents. Before each simulation session the pilots were given a briefing about the DA which included the procedures for anticipating and flying turns precisely and for executing the profile descents that were to be issued by the controllers. They were also briefed on the wind and weather conditions. Three wind conditions were tested: (1) calm (0 wind); (2) a direct tailwind of 70 knots at $35,000 \mathrm{ft}$, decreasing linearly to 0 knots at sea level; and (3) a direct headwind of 70 knots at $35,000 \mathrm{ft}$, decreasing linearly to 0 knot at sea level. For each simulation run, the aircraft was initialized on one of four primary arrival routes to Denver's northwest feeder fix, Drako, at altitudes of $29,000-35,000 \mathrm{ft}$ and a cruise speed of Mach 0.8 (see Figure 3). After the simulator was released into the ATC simulation, the pilots were asked to contact Denver Center, just as they normally would, and to maintain standard navigation and ATC procedures for a descent into Denver's Stapleton Airport. The only change to current procedures for a descent to the Drako feeder fix was that the crossing restriction at Drako was changed from "cross Drako between 17,000 ft and Flight Level 230 at 250 knots" to "cross Drako at 17,500 ft and descent speed." As the aircraft approached the Drako feeder fix, the air traffic controller issued the pilot an advisory in the form of a top-of-descent point and descent speed.

In order to have a basis for comparison, some of the pilot crews were asked to plan and fly a profile descent to Drako using the current standard operating procedure for their airline. All of the pilots flew a Mach 0.8/280 knots indicated airspeed 


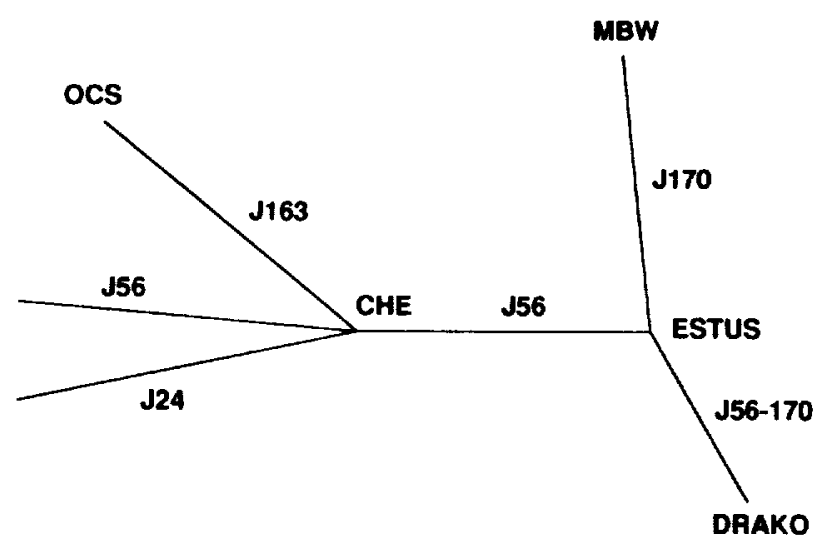

DEN

Fig. 3 Simulated arrival routes to Denver.

(KIAS) descent-speed profile. After this initial baseline descent, the pilots were asked to follow the advisories issued by the controllers in the simulated ATC environment. After each descent was completed, the aircraft was reinitialized on one of the four arrival routes to Drako and reinstated into the ATC simulation for another descent. At the conclusion of the 4-hr simulation session, each pilot was interviewed about the operational aspects of executing advisor-assisted profile descents.

\section{RESULTS}

Comparisons of actual aircraft states with the predicted 4D trajectories were the primary measures of performance. These states included altitude, position, time, CAS, Mach number, vertical speed, EPR, and total thrust. At the feeder fix, the aircraft were predicted to be at $17,500 \mathrm{ft}$, with descent speed and scheduled time depending on the wind conditions and the controller inputs. A total of 96 advisor-assisted descents were used for the statistical data base and can be broken into three categories for the purposes of comparison: fast (Mach 0.84/ $350 \mathrm{KIAS}$ ), nominal (Mach 0.8/320 KIAS), and slow (230 KIAS). In addition, 12 baseline descents were flown in which the pilots executed their current standard-airlineoperating-procedure profile descent (Mach 0.8/280 KIAS). Descents flown in the simulation at speeds other than these are not considered here since these three categories represent most descents, span the entire 727 descent-speed envelope, and provide a large enough statistical base from which to draw conclusions.

Figures 4-7 show the composite altitude profiles for the baseline, nominal, fast, and slow descents in calm wind conditions. Note that for the baseline runs, although all of the pilots were flying the same profile descent (Mach 0.8/280 KIAS), the range of top-of-descent points chosen by the pilots varied by as much as $25 \mathrm{n}$.mi. This is because pilots have their own simple "rules of thumb" for choosing a top-of-descent point. There are typically even greater variations in altitude profiles and arrival times for baseline descents when various wind conditions are present (2). Also note that for the slow descents, one aircraft began its descent 10 n.mi. earlier than necessary. In this descent, the pilot thought the procedure was to reduce speed early, so that the aircraft was already at 230 knots at the commanded top-of-descent point instead of beginning to slow at that point.

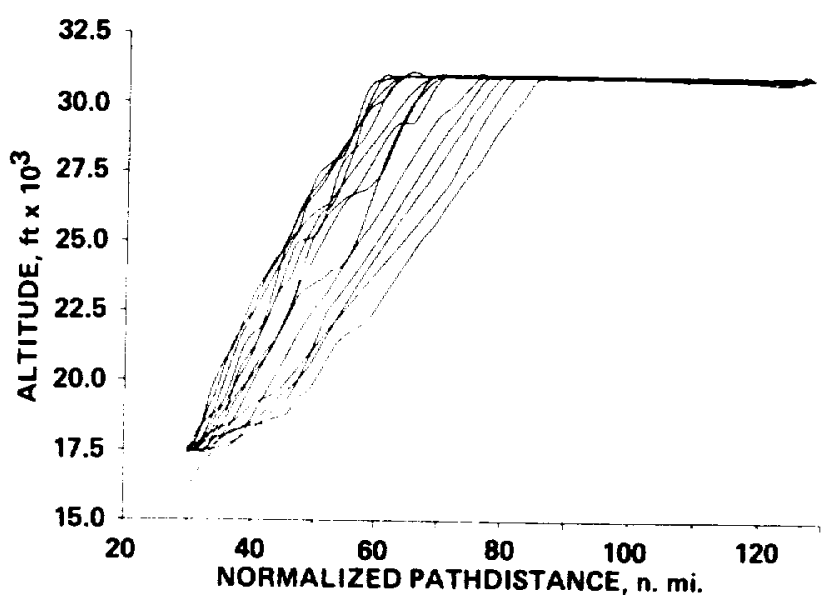

Fig. 4 Composite of all baseline descents flown in simulation.

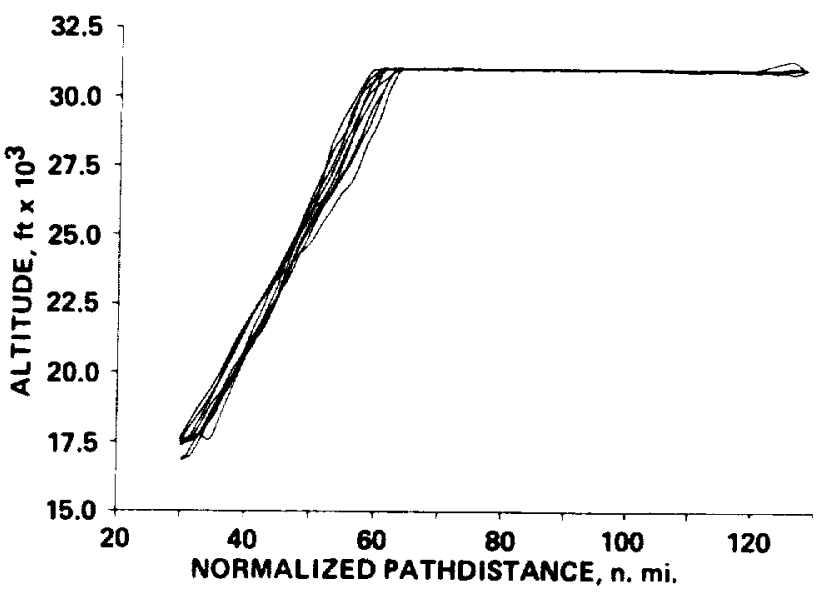

Fig. 5 Composite of all advisor-assisted descents (nominal) flown in simulation.

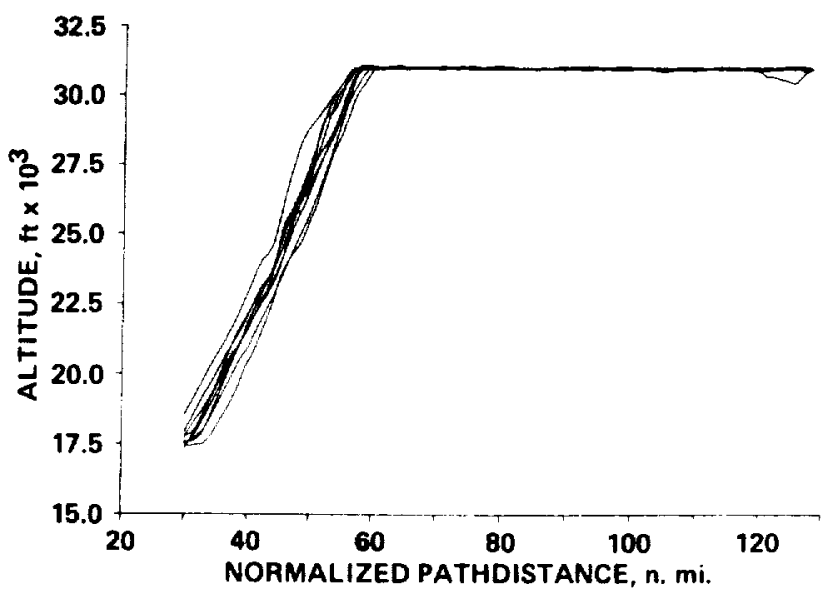

Fig. 6 Composite of all advisor-assisted descents (fast) flown in simulation. 


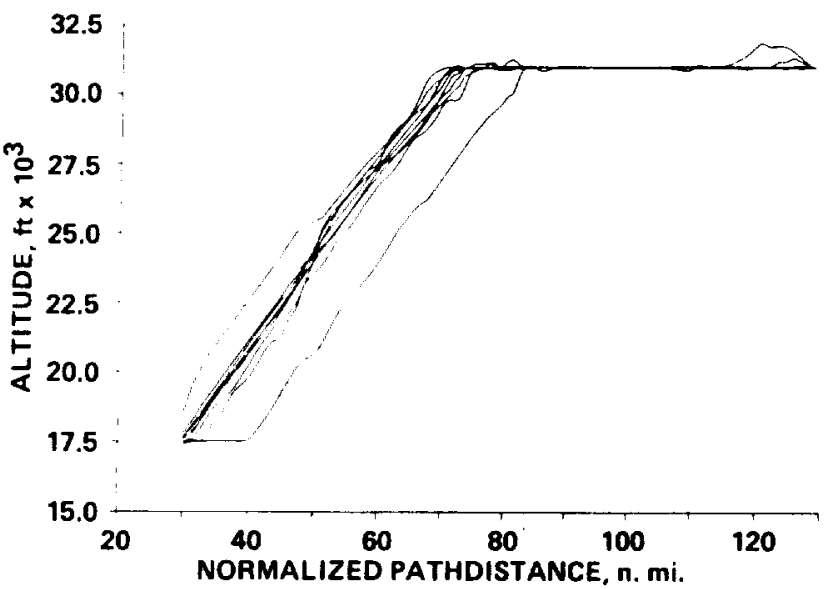

Fig. 7 Composite of all advisor-assisted descents (slow) flown in simulation.

Figure 8 shows arrival-time errors at feeder fix Drako for aircraft flying advisor-assisted descent-speed profiles for all three wind conditions. The mean arrival-time error was $13.4 \mathrm{sec}$ late, with a standard deviation of $15.6 \mathrm{sec}$. This histogram shows that most aircraft arrived within $\pm 20 \mathrm{sec}$ of their scheduled arrival time.

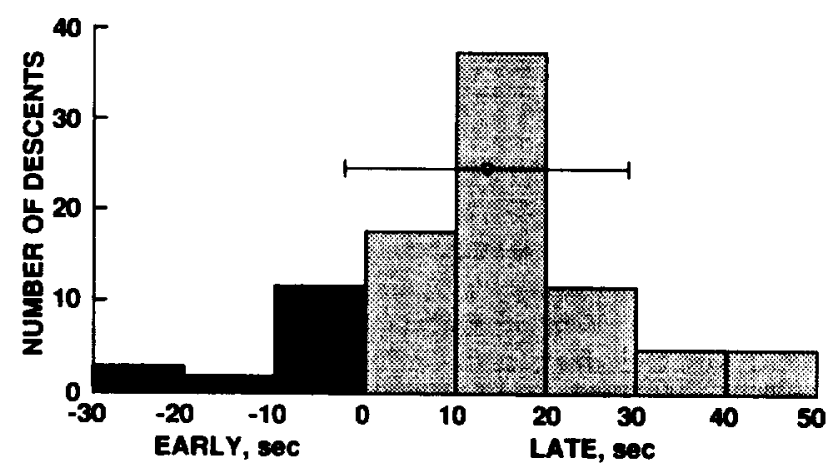

Fig. 8 Arrival-time errors for all three wind conditions (calm, headwind, and tailwind) at feeder fix Drako.

The 13.4-sec-late bias in arrival time can be traced to the manner in which turns are modeled by the DA algorithm. The DA assumes that the pilot will initiate the turn before arriving at a turn waypoint that is at the intersection of two VHF omnidirectional radio range (VOR) radials (such as Estus) shown in Figure 9. Although they were briefed to anticipate and fly the turns precisely, most pilots initiated the turn at the turn waypoint as shown in Figure 10. This has the effect of making the path flown by the pilot longer than the path modeled by the DA, and thus the aircraft would be expected to arrive at the feeder fix slightly behind schedule.

If the time errors that were accumulated during the turn are subtracted out of the arrival-time errors at Drako, one can readily see the effect of the turn on arrival-time accuracy. Figure 11 shows arrival-time errors, in 10-sec intervals, for advisorassisted descents in the case of calm winds. The pairs of histograms show the arrival-time errors with and without the turngenerated time errors included. By taking the turn-generated time error out, the mean arrival time for the calm-wind case is reduced from $13 \mathrm{sec}$ late to $4.6 \mathrm{sec}$ late, and the standard deviation is reduced from $16.5 \mathrm{sec}$ to $12.4 \mathrm{sec}$. These results more closely reflect those obtained in an earlier study of straight-in descents in which the mean arrival-time error was $6.1 \mathrm{sec}$ late and the standard deviation was $13 \mathrm{sec}(2)$. These data suggest that the DA predictive capability can potentially be raised so that descents with turns can be predicted as well as the straightin descents can be, either by modeling turns as pilots currently fly them or by having controllers issue turn advisories to match the turn prediction used in the current version of the DA algorithm.

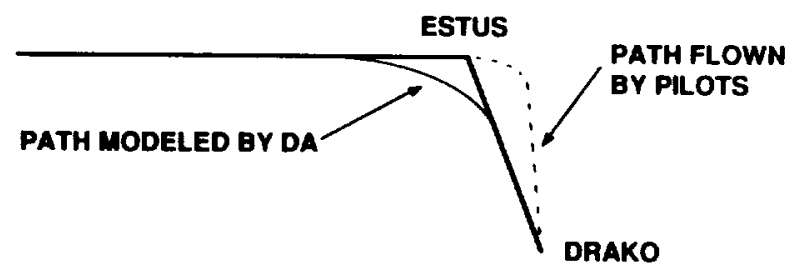

Fig. 9 Turns modeled by descent advisor.

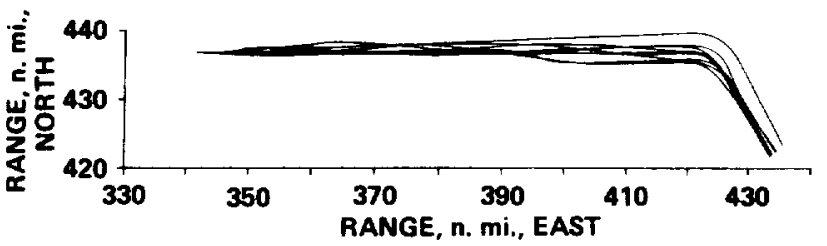

Fig. 10 Composite of horizontal paths for nominal $(0.8 / 320)$ descents in calm winds.

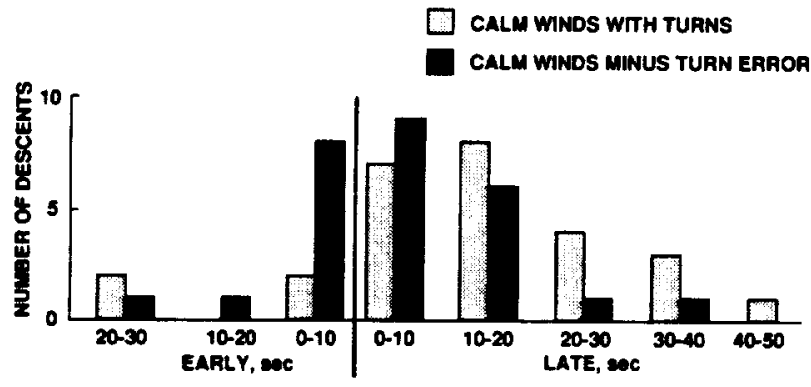

Fig. 11 Arrival-time errors with and without compensation for turn errors.

Table 1 lists the total variability in arrival time, defined as the difference between the earliest and latest arrival, at the feeder fix for the three wind conditions, and Table 2 lists the one-sigma standard deviation of arrival times. The nominal advisor-assisted descent in calm wind conditions most closely resembles the baseline descent and shows considerable improvement in both arrival-time variability and standard deviation. The fast and slow advisor-assisted descents show more error than the nominal in arrival-time accuracy, but this was to be expected since most pilots are not accustomed to flying profile descents at these speeds. In addition, the fast profile requires a high descent rate, which makes precise speed control more difficult. 
Table 1. Total variability of arrival time in seconds

\begin{tabular}{|l|c|c|c|c|}
\hline \multirow{2}{*}{ Wind condition } & \multirow{2}{*}{ Baseline } & \multicolumn{3}{|c|}{ Advisor-assisted } \\
\cline { 3 - 5 } & & Nominal & Fast & Slow \\
\cline { 3 - 5 } & & & & \\
Tailwind & & 40 & 47 & 69 \\
Headwind & & 48 & 79 & 33 \\
Calm & 87 & 37 & 72 & 54 \\
\hline
\end{tabular}

Table 2. One-sigma standard deviation of arrival time in seconds

\begin{tabular}{|l|c|c|c|c|}
\hline \multirow{2}{*}{ Wind condition } & \multirow{2}{*}{ Baseline } & \multicolumn{3}{|c|}{ Advisor-assisted } \\
\cline { 3 - 5 } & & Nominal & Fast & Slow \\
\cline { 3 - 5 } & & 11 & 12 & 21 \\
Tailwind & & 14 & 21 & 10 \\
Headwind & & 12 & 20 & 18 \\
Calm & 25 & & & \\
\hline
\end{tabular}

There are important parameters other than arrival time at the feeder fix that affect the success of a time-based trafficmanagement system. Aircraft should arrive at the feeder fix not only at their scheduled time, but at their scheduled altitude and speed. If aircraft arrived at the feeder fix on time, but with large altitude and speed errors, time errors and spacing errors would eventually become evident and propagate into the final approach area. The errors in the altitude and speed at the feeder fix were computed for the two most similar descents, the baseline descent and the advisor-assisted nominal descent in calm winds. For the baseline descents, the mean altitude error was $-131 \mathrm{ft}$ and the standard deviation was $389 \mathrm{ft}$. The advisorassisted descent had a mean altitude error of $-155 \mathrm{ft}$ and a standard deviation of $292 \mathrm{ft}$. Thus, the baseline and advisorassisted descents have essentially the same altitude error, and both are considered acceptable. This is not the case with airspeed, where the baseline descents resulted in a mean airspeed error of 6 knots and a standard deviation of 16 knots. The advisor-assisted nominal descents had a mean airspeed error of -5 knots and a standard deviation of 5 knots. The 16-knot standard deviation in airspeed for the baseline descents is not acceptable because it will rapidly propagate into significant time errors as the aircraft passes the current time control point and enters the Terminal Radar Approach Control (TRACON) airspace. Improvement to a 5-knot standard deviation in the advisor-assisted descents is more conducive to the precise scheduling required for time-based traffic management extending into the TRACON.

In the early stages of a simulation run, several pilots were skeptical about the utility of the DA tool. These pilots felt that they could achieve scheduled arrival times without advisor assistance. Therefore, some pilots were issued a time-only descent instruction (e.g., "Arrive at Drako at XX minutes and YY seconds past the hour"). Although they were able to meet the times accurately, the methods they used included extensive path-stretching, extended level flight at low altitudes, and excessive descent rates. Most of the advisor-assisted descents (and all of the descents used for statistical purposes) were issued in a conventional manner (i.e., top-of-descent point and descent speed). By the end of the 4-hr simulation session in which the pilots executed several advisor-assisted descents plus their own baseline descent in the ATC environment, nearly all pilots expressed enthusiastic support for the DA concept. They liked the DA because they were able to fly the descents with little or no training, and they were impressed with the accuracy in time, altitude, and speed at which they arrived at the feeder fix. The pilots also made several suggestions for improvement. They recommended (1) that the fast descents be restricted to good weather conditions, because of problems with turbulence and weather penetration; and (2) that provisions for cruise speed variations be incorporated, because many pilots would become frustrated with the slow descents. The algorithm has been modified to address these issues.

\section{CONCLUDING REMARKS}

A ground-based, 4D, DA algorithm has been developed and tested in a simulated ATC environment. The concept shows considerable promise for assisting air traffic controllers in meeting precise time schedules and spacing requirements. Simulation results showed that most pilots executing advisorassisted descents arrived at the feeder fix within $\pm 20 \mathrm{sec}$ of their scheduled arrival time, which is necessary if a time-based traffic management system is to be effective. The simulation also showed a late bias in arrival time for advisor-assisted descents, which may be attributed in large part to the method in which turns are modeled by the DA algorithm. This bias can be eliminated by changing the turn model to match more closely the turns executed by airline pilots. It is also critical in a timebased traffic management system that aircraft arrive at the feeder fix at the predicted altitude and speed. The simulation showed that the altitude error at the feeder fix was acceptable both for the advisor-assisted descents and for unassisted descents, but airspeed error was too large for the baseline descents. Advisor-assisted descents reduced the error to acceptable levels. Pilots were able to execute the advisorassisted descents without prior training and were enthusiastic about the DA. Controller evaluations of the DA concept and interface were also positive, and indicated considerable promise for this tool. Current plans call for additional simulation evaluations of enhancements to the DA algorithm and controller interface. If these tests are successful, a live operational evaluation of the DA at an enroute traffic control center is anticipated.

\section{REFERENCES}

(1) Erzberger, H. and Nedell, W., "Design of Automation Tools for Management of Descent Traffic," NASA TM-101078, Dec. 1988.

(2) Green, S.M., Davis, T.J., and Erzberger, H., "A Piloted Simulator Evaluation of a Ground-Based 4D Descent Advisor Algorithm," Proceedings of the 1985 AlAA Coliference on Guidance, Navigation and Control, Monterey, CA, pp. 1173-1180, Aug. 1987. 
(3) Tobias, L., Erzberger, H., and Volckers, U., "Controller Evaluations of the Descent Advisor Automation Aid," AIAA Guidance, Navigation and Control Conference Paper $\# 89-3624$, Boston, Aug. 1989. (Note: this conference)
(4) Erzberger, H. and Chapel, J., "Ground Based Concept for Time Control of Aircraft Entering the Terminal Area," Proceedings of the 1985 AIAA Conference on Guidance, Navigation and Control, Snowmass, CO, pp. 301-306, Aug. 1985. 


\begin{tabular}{|c|c|c|}
\hline \multicolumn{3}{|c|}{ Report Documentation Page } \\
\hline $\begin{array}{l}\text { 1. Report No. } \\
\text { NASA TM-10108. }\end{array}$ & 2. Government Accession No. & 3. Recipient's Catalog No. \\
\hline \multicolumn{2}{|l|}{ 4. Title and Subtitle } & $\begin{array}{l}\text { 5. Report Date } \\
\text { June } 1989\end{array}$ \\
\hline \multicolumn{2}{|c|}{$\begin{array}{l}\text { Piloted Simulation of a Ground-Based Time-Control Concept } \\
\text { for Air Traffic Control }\end{array}$} & 6. Performing Organization Code \\
\hline \multicolumn{2}{|l|}{ 7. Author(s) } & $\begin{array}{l}\text { 8. Performing Organization Report No. } \\
\text { A-89085 }\end{array}$ \\
\hline \multicolumn{2}{|c|}{ Thomas J. Davis and Steven M. Green } & 10. Work Unit No. \\
\hline \multicolumn{2}{|c|}{ 9. Performing Organization Name and Address } & $505-67-21$ \\
\hline \multirow{2}{*}{\multicolumn{2}{|c|}{$\begin{array}{l}\text { Ames Research Center } \\
\text { Moffett Field, CA } 94035\end{array}$}} & 11. Contract or Grant No. \\
\hline & & 13. Type of Report and Period Covered \\
\hline \multicolumn{2}{|c|}{ 12. Sponsoring Agency Name and Address } & Technical Memorandum \\
\hline \multicolumn{2}{|c|}{$\begin{array}{l}\text { National Aeronautics and Space Administration } \\
\text { Washington, DC 20546-0001 }\end{array}$} & 14. Sponsoring Agency Code \\
\hline \multicolumn{3}{|c|}{ 15. Supplementary Notes } \\
\hline \multicolumn{3}{|c|}{$\begin{array}{l}\text { Point of Contact: Thomas J. Davis, Ames Research Center, MS 210-9, Moffet Field, CA } 94035 \\
(415) 694-5452 \text { or FTS } 464-5452 \\
\text { This paper was originally prepared for the AIAA American Control Conference, June } 1989\end{array}$} \\
\hline
\end{tabular}

A concept for aiding air traffic controllers in efficiently spacing traffic and meeting scheduled arrival times at a metering fix has been developed and tested in a real-time simulation. The automation aid, referred to as the ground-based four-dimensional descent advisor (DA), is based on accurate models of aircraft performance and weather conditions. The DA generates suggested clearances, including both topof-descent-point and speed-profile data, for one or more aircraft in order to achieve specific time or distance separation objectives. The DA algorithm is used by the air traffic controller to resolve conflicts and issue advisories to arrival aircraft. A joint simulation was conducted using a piloted simulator and an advanced-concept air traffic control simulation to study the acceptability and accuracy of the DA automation aid from both the pilot's and the air traffic controller's perspectives. This paper focuses on the results of the piloted simulation. In the piloted simulation, airline crews executed controller-issued descent advisories along standard curved-path arrival routes, and were able to achieve an arrival-time precision of $\pm 20 \mathrm{sec}$ at the metering fix. An analysis of errors generated in turns resulted in further enhancements of the algorithm to improve the predictive accuracy. Evaluations by pilots indicate general support for the concept and provide specific recommendations for improvement. Operational issues concerning how the DA was used for prediction, intrail spacing, and metering in a multiaircraft environment are described in a companion paper (Tobias; see block 15).

17. Key Words (Suggested by Author(s))

Air traffic control

Descent advisor

Profile descents

Time-based traffic management
18. Distribution Statement

Unclassified-Unlimited

Subject Category: 03
19. Security Classif. (of this report)

Unclassified

\author{
20. Security Classif. (of this pagel \\ Unclassified
}

$\left[\begin{array}{c|r}21 . \text { No. of pages } & 22 . \text { Price } \\ 9 & \mathrm{A02}\end{array}\right.$



$-$ 